\title{
A multispecies investigation of the strain rate sensitivity of the modulus of cortical bone
}

\author{
Claire Victoria Rampersadh ${ }^{1}$, Lee-Anne Welgemoed ${ }^{1}$, and Trevor John Cloete ${ }^{1^{*}}$ \\ ${ }^{1}$ Blast Impact and Survivability Research Unit (BISRU), Department of Mechanical Engineering, \\ University of Cape Town, South Africa
}

\begin{abstract}
The stiffness of cortical bone shows both inter- and intra-species variation. Currently, it is unclear whether this variation is due to differing testing protocols or an inherent feature of the material. Additionally, there is a lack of literature dealing with species other than human and bovine, particularly in the intermediate strain rate regime. In this study, cortical bone specimens were machined from the femurs of four species: baboon, crocodile, sheep and ostrich. Specimens were tested in the quasi-static and intermediate strain rate regimes using consistent testing protocols implemented by a single researcher. The results show a similar strain rate dependence for all species, i.e. the modulus shows negligible rate effects in the quasi-static regime, but a significant increase when moving to intermediate strain rates. This suggests that while the stiffness of the bone is species dependent, the effect of strain rate may be species independent. The observed intra- and inter-species variation is less than that reported in literature, highlighting the importance of a consistent testing protocol in multi-species studies.
\end{abstract}

\section{Introduction}

Cortical bone is a strain rate dependent, viscoelastic-quasibrittle material [1] found throughout the body and particularly in the diaphysis of long bones. It has a densely packed microstructure that varies both across and within species [2]. Previous research has shown that the mechanical properties of cortical bone are affected by several factors, including the bone microstructure [3], as well as specimen storage methods and mediums [4]. The latter makes the comparison of properties between different studies particularly difficult as it is unclear whether the variations are due to inherent differences in the bone or differences due to experimental protocol.

The majority of research on the mechanical properties of cortical bone has focused on human and bovine bone [5], with other species being poorly represented in the literature. Multiple species studies are rare [2,6] and typically contain data collected over several independent test series. Multiple species are seldom included in a single test series, making it difficult to distinguish between inherent variations in the properties of species and those caused by differing storage or testing methods. Furthermore, the studies do not consider the intermediate strain rate regime, which is where many dynamic bone fractures occur [1].

\footnotetext{
${ }^{*}$ Corresponding author: trevor.cloete@uct.ac.za
} 
There appears to be a general lack of higher strain rate data for species other than human and bovine in the literature. Most studies present apparent modulus results obtained at a quasi-static strain rate [2,7] or reported with no associated strain rate, either due to lack of reporting $[6,8]$ or due to the use of testing methods that do not correlate with particular strain rates [9]. Additionally, until work by Adharapurapu et al. [10], no concerted effort had been made to keep the strain rate near constant during testing. As cortical bone is a strain rate dependent material, a near constant strain rate during testing is important to produce more accurate material properties. Adharapurapu et al. used a Split Hopkinson Bar (SHB) in conjunction with a sacrificial pulse shaper. An alternative technique was employed in work by Cloete et al. [1], who developed the Cone-in-Tube (CiT) striker to shape the pulse.

This paper presents the results of a study intended to compare the strain rate dependence of the mechanical properties of cortical bone across multiple species in a consistent manner by minimising experimental variation through the concurrent storage, preparation and testing of all specimens from all species by a single researcher using the same equipment.

\section{Materials and methods}

\subsection{Specimen machining}

Four species were selected for investigation, namely baboon, crocodile, sheep and ostrich. This choice represents three classes (Mammalia, Reptilia, and Aves) and four distinct orders (Primates, Crocodilia, Artiodactyla, and Struthioniformes). Consequently, the large range of body plans, developmental histories and locomotion modes is expected to provide the greatest possible inherent variation in cortical bone properties of terrestrial vertebrates.

Frozen ostrich and sheep femurs were obtained from local butcheries. The crocodile femurs were obtained from a crocodile farm, where a crocodile had succumbed to cold weather. Frozen baboon bones were provided by the Department of Forensics Science at the University of Cape Town. After collection, all bones were stored as whole bones, wrapped in plastic at $-32^{\circ} \mathrm{C}$ for one year prior to testing.

Prior to machining, each bone was removed from the freezer and allowed to thaw. Cylindrical slices of bone were cut from the mid-diaphysis and longitudinal strips of cortical bone were excised from the cut slices. The cortical bone strips were machined into smooth cylinders on a lathe and sectioned to provide small, cylindrical cortical bone specimens. It was found that the cortical bone layer was very thin in the baboon, crocodile and sheep bone, hence these specimens were machined to $2.5 \mathrm{~mm}$ in diameter and $3 \mathrm{~mm}$ in height, whereas the ostrich specimens were machined to $3 \mathrm{~mm}$ in diameter and $4 \mathrm{~mm}$ in height. After machining, specimens were stored in natural saline solution in a bio-freezer at $-32^{\circ} \mathrm{C}$.

When required for testing, specimens were removed from the freezer and thawed at room temperature. Once thawed, the specimens were removed from the saline solution and dabbed dry using paper towel before commencing with the testing procedure.

\subsection{Quasi-static testing}

Quasi-static compression testing was performed on an Instron quasi-static testing machine using a $5 \mathrm{kN}$ load cell at strain rates of $10^{-3} \mathrm{~s}^{-1}, 10^{-2} \mathrm{~s}^{-1}$ and $10^{-1} \mathrm{~s}^{-1}$. Three specimens from each species were tested at each strain rate, for a total of 36 quasi-static tests. Equal numbers of specimens of each species were tested in random order on each testing day, by the same researcher on the same machine to avoid introducing experimental bias into the data. The data obtained from the Instron tester was processed as described by Welgemoed [11], which included compliance correction and linearisation and apparent moduli were recorded. 


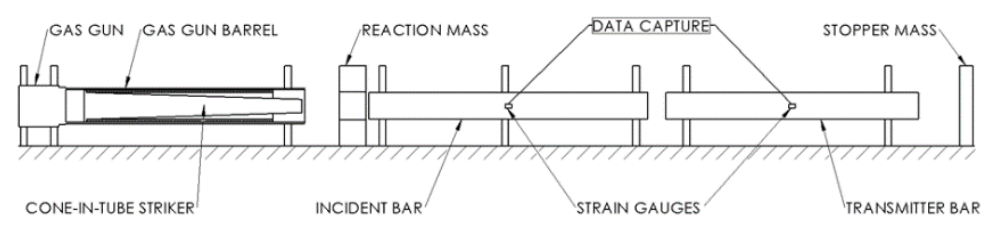

Figure 1: Schematic of a CiT striker in a typical SHB setup.
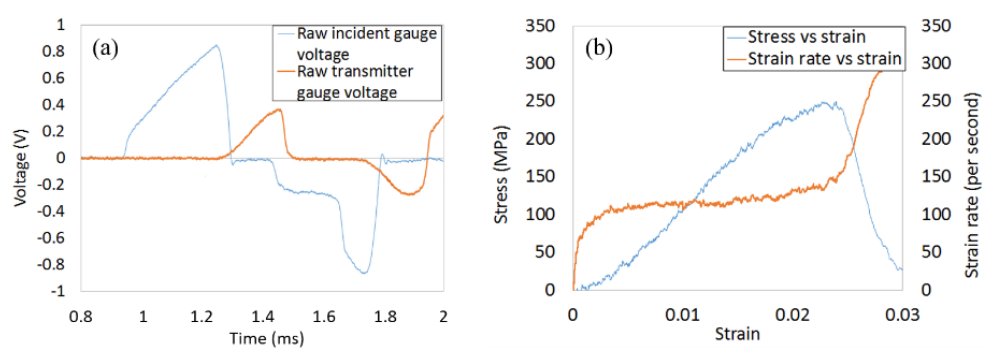

Figure 2: Examples of (a) a raw SHB signal showing voltage over time, and (b) the final processed SHB signal showing the constant strain rate obtained using a CiT striker.

\subsection{Intermediate strain rate testing}

A Split Hopkinson Bar incorporating a CiT striker, shown in Figure 1, was employed to test the cortical bone specimens at strain rates between $45 \mathrm{~s}^{-1}$ and $297 \mathrm{~s}^{-1}$, i.e. the intermediate strain rate regime. The CiT striker [1] was used to produce an increasing stress pulse which allows for constant strain in the specimen. The increasing stress pulse is controlled by the slope of the cone and can be matched to the apparent modulus of a strain hardening material. This provides a near constant strain rate during a test, as shown in Figure 2.

Three cone angles were chosen based on the apparent modulus results measured during the quasi-static testing series. A numerical model [1] was used to predict the wave generated by each conical striker and the minimum diameter of the cone was adjusted until the gradient of the obtained wave was equivalent to the estimated apparent modulus. The maximum diameter and length of the cones were kept constant at $24 \mathrm{~mm}$ and $850 \mathrm{~mm}$, respectively, to allow the cones to fit into the available gas gun barrel. Minimum diameters for the low, midrange and high moduli cones were set to be $6.5 \mathrm{~mm}, 7.4 \mathrm{~mm}$ and $8.3 \mathrm{~mm}$, respectively.

Five cortical bone specimens from each species were tested in the intermediate strain rate regime, with the exception of the crocodile, for which eight specimens were tested. The data from the SHB was processed using wave theory [1] to obtain the strain rate, strain and stress within the specimen. The stress-strain curves were used to obtain the apparent moduli.

\section{Results}

Figure 3 shows the apparent modulus results obtained from the quasi-static and intermediate strain rate compression testing for all species. Although there is some overlap in the data, there is clear separation between the different species and patterns are easily identified across the various species and strain rates. The data shows that, on average, the crocodile bone displays the lowest apparent modulus, followed by the sheep bone, ostrich bone and, finally, baboon bone, which displays the stiffest behaviour of the tested species. There is also a clear pattern in strain rate dependence across the species. In general, the species showed little to no strain rate dependence in the quasi-static regime, but a distinct increase in stiffness when transitioning to the intermediate strain rate regime. The increase from the quasi-static to 


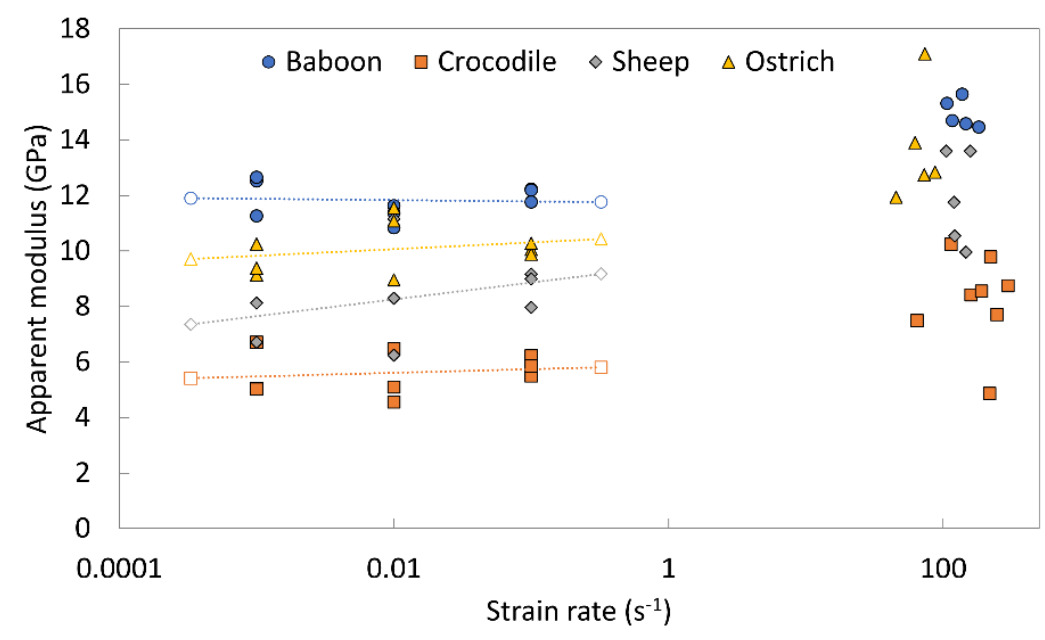

Figure 3: Comparison of apparent moduli for all species across all strain rates tested.

intermediate strain rate regimes was calculated as $3.1 \mathrm{GPa}(26 \%), 2.6 \mathrm{GPa}(47 \%), 4.0 \mathrm{GPa}$ $(50 \%)$ and $3.6 \mathrm{GPa}(36 \%)$ for the baboon, crocodile, sheep and ostrich bone, respectively.

The data in Figure 3 also shows a clear difference in the amount of scatter between species and across strain rates. In the crocodile, sheep and ostrich bone, the scatter in the results was low to moderate in the quasi-static tests and significantly higher in the intermediate strain rate tests. The baboon bone showed the least amount of scatter overall, including in intermediate strain rate results, which showed no significant increase in the amount of scatter.

\section{Discussion}

\subsection{Interspecies and variation}

Figure 3 shows that there is no significant difference between the range of values reported for a single species across the three quasi-static strain rates. However, a clear and distinct increase in moduli across all species may be observed when moving from the quasi-static to intermediate strain rate regime, with all species showing absolute changes in the range of 2.64.0 GPa. Previous work investigating bovine bone showed a similar increase of approximately $5.5 \mathrm{GPa}(55 \%)$ [1]. These results indicate that while the cortical bone stiffness is species dependent, the effect of strain rate on the stiffness is relatively consistent across all species tested. As these species were chosen to represent the widest range of cortical bone stiffnesses available, it may be theorised that similar strain rate dependence will be found in other species, although more testing is required to draw definitive conclusions.

The crocodile, sheep and ostrich bone showed the largest amount of scatter in the intermediate strain rate results. This may be attributed to the fact that the higher strain rate testing is likely to highlight any flaws in the material or imperfections in specimen manufacture, due to the more aggressive nature of the test. As a result, the increase in scatter could be caused by the presence of microdamage in some specimens, a difference in microstructure, changes in porosity, etc.

A factor of particular interest when viewing these results is the density of the bone specimens. As shown in Figure 4, there is no discernible trend between the intra-species densities of the specimens and the measured moduli. The inter-species moduli appear to show some correlation to the density in that the average density correlates reasonably well to the 


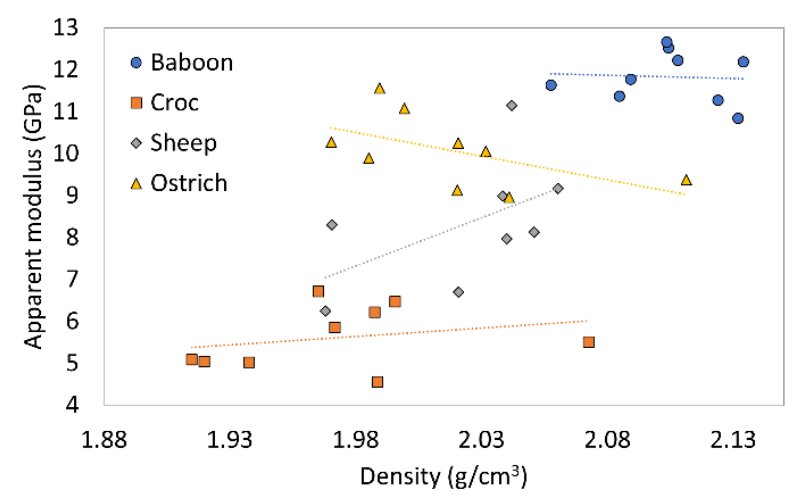

Figure 4: Graph showing the variation of apparent modulus with density.

average modulus for the baboon and crocodile bone, i.e. the baboon specimens have both the highest average density and the highest average modulus. The sheep and ostrich bone have near equivalent average densities, however this could be due to the small sample size of the data. The reason for the inter-species trend without a similar intra-species trend requires further investigation.

\subsection{Comparison to literature}

As mentioned in the introduction, studies investigating the mechanical properties of cortical bone from species other than human and bovine are rare. This section presents a comparison of the results of this work with the sparse literature that was found for the species tested.

Baboon: Wang et al. [9] investigated baboon bone using ultrasonic testing at 5 locations in the skull and reported apparent moduli ranges of 9.2-12.2 $\mathrm{GPa}\left(\mathrm{E}_{1}\right), 13.6-18.0 \mathrm{GPa}\left(\mathrm{E}_{2}\right)$ and 17.2-25.7 $\mathrm{GPa}\left(\mathrm{E}_{3}\right)$. The quasi-static data captured in this work (10.8-12.7 GPa) falls mostly within the lower range reported by Wang et al., whereas the intermediate strain data (14.5-15.7 GPa) falls within the mid-range reported by Wang et al. Both the quasi-static and intermediate data show less variation than the range of values reported by Wang et al. [9].

Crocodile: Currey studied crocodile [6] and alligator [2] bone. In the crocodile study [6], Curry reported apparent moduli in the range of approximately 6-8.5 GPa, with no description being given with regards to testing protocol or sampling location. In the alligator study [2], an apparent modulus of $13.0 \mathrm{GPa}$ at a strain rate of $0.2 \mathrm{~s}^{-1}$ was reported for specimens taken from the femur. The quasi-static results in this work (4.56-6.71 GPa) fall below and on the lower end of the range reported by Currey for crocodile bone [6], whereas the intermediate results (4.87-10.2 GPa) fall, for the most part, on the upper end of the Currey crocodile range [6]. All the results fall well below the $13 \mathrm{GPa}$ reported for alligator bone [2].

Sheep: Spatz et al. [7] reported apparent moduli for sheep bone as 26-31.5 GPa. Specimens in the Spatz study were obtained from long bones and tested at a quasi-static strain rate (strain rate unknown). The apparent moduli obtained in this work at both quasi-static (4.74-11.2 GPa) and intermediate strain rates (9.95-13.6 GPa) were all significantly lower than values reported by Spatz et al. [7]. However, considering that apparent moduli for human and bovine bone generally fall in the range of 5-25 GPa [5], the Spatz values appear to be exceptionally high. Nevertheless, these appear to be the only literature values available for sheep bone and further testing is required to confirm.

Ostrich: Two studies have reported moduli of ostrich bone using compression testing. Yamada and Evans [8], who did not provide details on strain rate or sampling locations, reported a value of 5.30 GPa. Cuff et al. [12] reported a value of 5.03 GPa obtained using 
nanoindentation on samples from the skull. Both the quasi-static (8.96-11.6 GPa) and intermediate strain rate results $(11.9-17.1 \mathrm{GPa})$ in this work fall above the literature values.

With the exception of the ostrich bone, the apparent moduli obtained in this work fall at the lower end or below the values reported in literature. This may be due to an ageing effect as discussed in [11]. Other possible reasons for the differing values in this work and the literature may be due to samples being extracted from different body parts, different testing techniques or a lack of reported strain rate in the literature. Overall, the inter-species data obtained in this work shows modest variation compared to that reported in the literature.

\section{Conclusions and recommendations}

This study examines the apparent moduli of cortical bone specimens across multiple species and strain rates in the quasi-static and intermediate regime. An important part of the study was the care that was taken to keep specimen manufacture, storage protocols and testing methods consistent between species so as to avoid introducing experimental bias.

The results of the study showed a distinct separation between the responses of the different species, with crocodile bone showing the most compliant behaviour and baboon bone showing the stiffest behaviour. Additionally, the variation of results within species like the baboon, sheep and ostrich was much smaller than the range of values reported in literature, suggesting that the increased range may be due to variation in testing protocols, as opposed to increased variability in the material.

The apparent moduli for all species showed a clear strain rate dependence, with minimal difference in the quasi-static regime and a distinct increase in the intermediate strain regime. This consistent response highlights the importance of consistent testing protocols, specimen machining and storage techniques in cortical bone research.

\section{Acknowledgements}

The financial assistance of the National Research Foundation and the University of Cape Town is hereby acknowledged. Opinions expressed and conclusions arrived at are those of the authors and are not necessarily to be attributed to the National Research Foundation.

\section{References}

1. T. J. Cloete, G. Paul, E. B. Ismail, Phil. Trans. R. Soc. A 372, 2015 (2014).

2. J. D. Currey, J. Biomech. 37, 549 (2004).

3. J. D. Currey, J Biomech 36, 1487 (2003).

4. B. Kaye, C. Randall, D. Walsh, P. Hansma, Open Bone J. 4 (2012).

5. T. P. M. Johnson, S. Socrate, M. C. Boyce, Acta Biomater. 6, 4073 (2010).

6. J. Currey, Am. Zool. 24, 5 (1984).

7. H. Spatz, E. O'Leary, J. F. Vincent, Proc. R. Soc. Lond. B Biol. Sci. 263, 287 (1996).

8. H. Yamada, F. G. Evans, Strength of biological materials, Williams \& Wilkins, 1970.

9. Q. Wang, D. S. Strait, P. C. Dechow, J. Hum. Evol. 51, 375 (2006).

10. R. R. Adharapurapu, F. Jiang, K. S. Vecchio, Mater. Sci. Eng. C 26, 1325 (2006).

11. L.-A. Welgemoed, An experimental investigation of interspecies variation in mechanical properties of cortical bone, University of Cape Town, Cape Town, 2018.

12. A. R. Cuff, J. A. Bright, E.J. Rayfield, PeerJ, 1 (2015). 